\title{
ERGODIC THEORY AND BOUNDARIES
}

\author{
BY \\ M. A. AKCOGLU AND R. W. SHARPE
}

1. Introduction. Let $T$ be a conservative positive contraction on the $L_{1}$ space of a finite measure space $(X, \mathscr{F}, \mu)$. A theorem of Chacon [5], [2] shows that $T$ defines a sub $\sigma$-field $\mathscr{I}$ of $\mathscr{F}$, consisting of invariant subsets of $X$. The ratio ergodic limits are measurable with respect to $\mathscr{I}$ [5], [2] and the class of these limits contains $L_{\infty}(X, \mathscr{I}, \mu)$, which can be considered as the invariant functions of the adjoint transformation [2]. The main purpose of the present paper is to show that any positive contraction on $L_{1}(X, \mathscr{F}, \mu)$ behaves, asymptotically, like a conservative transformation (Theorems 3 and 4 ) and that the invariant functions of the adjoint transformation can be approximated by the ratio ergodic limits.

Intuitively, a ratio ergodic limit corresponds to the result of an averaging process of different values of a function. It is then natural to consider these limits as functions that are smooth with respect to the asymptotic behaviour of the transformation. This leads (Theorem 6) to a Martin-Doob type representation [12], [8] of invariant functions as the $L_{\infty}$ functions of a compact Hausdorff space $\mathscr{M}$ with a Baire measure. The topology on $\mathscr{M}$ is just strong enough to make the ergodic limits to correspond to continuous functions. As an example we consider a transformation of Feller [10] and show that for this case the above representation is identical with the Poisson representation of harmonic functions in the unit disk. We also consider the possibility of joining $X$ and $\mathscr{M}$, convergence of measures to $\mathscr{M}$ in $X \cup \mathscr{M}$ (Theorem 7), and a relation (Lemma 9) between the Feller and Martin-Doob type representations, corresponding to a result of Feldman [9].

2. Preliminaries. Let $(X, \mathscr{F}, \mu)$ be a finite measure space and let $L_{p}=$ $L_{p}(X, \mathscr{F}, \mu), 1 \leqq p \leqq \infty$ be the usual Banach spaces, and $L_{p}^{+}$denote the positive cone of $L_{p}$. Let $T: L_{1} \rightarrow L_{1}$ be a positive linear contraction and $U: L_{\infty} \rightarrow L_{\infty}$ be its dual. For $\alpha \in L_{\infty}$ define $T_{\alpha}: L_{1} \rightarrow L_{1}$ as $T_{\alpha} f=\alpha f+T(1-\alpha) f, f \in L_{1}$, and let $U_{\alpha}$ be its dual. If $\chi_{E}$ is the characteristic function of $E \in \mathscr{F}$ we write $T_{E}$ and $U_{E}$ instead of $T_{\chi_{E}}$ and $U_{\chi_{E}}$.

The following partial ordering of $L_{1}^{+}$is similar to that of Bishop and deLeeuw given in [3].

Definition 1. For $f, g \in L_{1}^{+}, f \prec g$ if and only if there exist an integer $n \geqq 1$ and $\alpha_{1}, \ldots, \alpha_{n} \in L_{\infty}$ such that $0 \leqq \alpha_{i} \leqq 1$ for $i=1, \ldots, n$ and $g=T_{\alpha_{n}} \cdots T_{\alpha_{1}} f$.

This relation is reflexive and transitive and $f \prec g$ implies $\|f\|_{1} \geqq\|g\|_{1}$. Also, an

Received by the editors March 13, 1967. 
induction argument shows that if $f \prec g$ then there exists an integer $n \geqq 1$ such that $g \prec T^{n} f$. Hence $\left\{g \in L_{1}^{+} \mid g \succ f\right\}$ is (upward) directed by $\prec$.

Definition 2. For $E \in \mathscr{F}, f \in L_{1}^{+}$let

$$
\Psi_{E} f=\sup _{g>f} \int_{E} g d \mu, \quad \Theta_{E} f=\lim _{g>f} \Psi_{E} g
$$

Note that $\Theta_{E} f=\lim _{n \rightarrow \infty} \Psi_{E} T^{n} f$.

Lemma 1. The limits $\psi_{E}=\lim _{n \rightarrow \infty} U_{E}^{n} \chi_{E}$ and $\theta_{E}=\lim _{n \rightarrow \infty} U^{n} \psi_{E}$ both exist (a.e.) and satisfy

$$
\Psi_{E} f=\int \psi_{E} f d \mu, \quad \Theta_{E} f=\int \theta_{E} f d \mu
$$

Proof. By induction, $U_{E}^{n} \chi_{E} \uparrow$ and $U^{n} \psi_{E} \downarrow$, so the limits exist. Now if $f \in L_{\infty}^{+}$ satisfies

$$
\chi_{E} U f \leqq \chi_{E} f, \quad \chi_{E^{c}} U f \geqq \chi_{E^{c}} f
$$

with $E^{c}=X-E$, then for all $\alpha \in L_{\infty}, 0 \leqq \alpha \leqq 1$, we have

$$
U_{\alpha} f=\alpha f+(1-\alpha) U f \leqq \chi_{E} f+\chi_{E} c U f=U_{E} f
$$

Since, by induction, $U_{E}^{n} \chi_{E}$ satisfies $\left(^{*}\right)$ for all $n \geqq 0$, we get, again by induction, $U_{\alpha_{n}} \cdots U_{\alpha_{1}} \chi_{E} \leqq U_{E}^{n} \chi_{E}$, and hence $\Psi_{E} f=\int \psi_{E} f d \mu$. The final part follows from the definition (cf. also [4] and [2]).

Definition 3. For $E, F \in \mathscr{F}$, let

$$
\psi_{E F}=\psi_{E}+\psi_{F}-\psi_{E \cup F}, \quad \theta_{E F}=\theta_{E}+\theta_{F}-\theta_{E \cup F} .
$$

$\Psi_{E F}, \Theta_{E F}$ are the functionals on $L_{1}$ defined by the $L_{\infty}$ functions $\psi_{E F}, \theta_{E F}$.

We note that $\psi_{E F}$ and $\theta_{E F}$ are monotone and subadditive in each index. This follows easily from the following general result, which will be useful to obtain other relations between these set functions (cf. [7]).

Lemma 2. If $a_{i}$ is real and $A_{i} \in \mathscr{F}$ for $i=1, \ldots, n$ and $A=\bigcup_{i=1}^{n} A_{i}$, then $\chi_{A} \sum_{i=1}^{n} a_{i} \psi_{A_{i}} \geqq 0$ implies $\sum_{i=1}^{n} a_{i} \psi_{A_{i}} \geqq 0$ and $\sum_{i=1}^{n} a_{i} \theta_{A_{i}} \geqq 0$.

Proof. If $f \in L_{1}^{+}$and $E \subset F, E, F \in \mathscr{F}$, then by induction: $\chi_{F}^{c} T_{F}^{n} f \leqq \chi_{F}^{c} T_{E}^{n} f$. Hence

$$
\begin{aligned}
0 \leqq \int_{F^{c}} \psi_{E} T_{F}^{n} f d \mu & \leqq \int_{F^{c}} \psi_{E} T_{E}^{n} f d \mu \leqq \int_{E^{c}} \psi_{E} T_{E}^{n} f d \mu \\
& \leqq \int \psi_{E} T_{E}^{n} f d \mu-\int_{E} \psi_{E} T_{E}^{n} f d \mu \leqq \Psi_{E} f-\int_{E} T_{E}^{n} f d \mu \rightarrow 0
\end{aligned}
$$


as $n \rightarrow \infty$. Now

$$
\begin{aligned}
\int \psi_{E} f d \mu & =\int \psi_{E} \chi_{F} f d \mu+\int \psi_{E} \chi_{F} c d \mu=\int \psi_{E} \chi_{F} f d \mu+\int \psi_{E} T_{E} \chi_{F}^{c} f d \mu \\
& =\int \psi_{E} \chi_{F} f d \mu+\int \psi_{E} T \chi_{F} c f d \mu=\int \psi_{E} T_{F} f d \mu \\
& =\int \psi_{E} T_{F}^{n} f d \mu=\int_{F} \psi_{E} T_{F}^{n} f d \mu+\int_{F^{c}} \psi_{E} T_{F}^{n} f d \mu,
\end{aligned}
$$

and hence $\lim _{n \rightarrow \infty} \int_{F} \psi_{E} T_{F}^{n} f d \mu=\int \psi_{E} f d \mu$. Using this for the case of $A_{i} \subset A$, $i=1, \ldots, n$, we get

$$
0 \leqq \sum_{i=1}^{n} a_{i} \int_{A} \psi_{A_{i}} T_{A}^{n} f d \mu \rightarrow \sum_{i=1}^{n} a_{i} \int \psi_{A_{i}} f d \mu
$$

as $n \rightarrow \infty$, which proves the first assertion. Since $U$ is positive, the remainder follows.

LEMMA 3. If $\chi_{E} \theta_{E} \geqq \alpha \chi_{E}$ then $\theta_{E} \geqq \alpha \psi_{E}$.

Proof. From the proof of the previous lemma we have that, for $f \in L_{1}^{+}$,

$$
\lim _{n \rightarrow \infty} \int_{E^{c}} \theta_{E} T_{E}^{n} f d \mu=0 .
$$

Hence

$$
\begin{aligned}
\int \theta_{E} f d \mu & =\int \theta_{E} T_{E}^{n} f d \mu=\lim _{n \rightarrow \infty} \int_{E} \theta_{E} T_{E}^{n} f d \mu \\
& \geqq \alpha \lim _{n \rightarrow \infty} \int_{E} T_{E}^{n} f d \mu \geqq \alpha \int \psi_{E} f d \mu .
\end{aligned}
$$

Finally we prove the following.

LeMma 4. For $E, F \in \mathscr{F},\left\|\theta_{E}\right\|_{\infty}=\left\|\chi_{E} \theta_{E}\right\|_{\infty}=0$ or 1 and $\left\|\theta_{E F}\right\|_{\infty}=\left\|\chi_{E} \theta_{E F}\right\|_{\infty}=$ $\left\|\chi_{F} \theta_{E F}\right\|_{\infty}=0$ or 1 .

Proof. For $g \in L_{1}^{+}$, as $n \rightarrow \infty, 0 \leqq \Theta_{E}\left(\chi_{E^{c}} T_{E}^{n} g\right) \leqq \Psi_{E}\left(\chi_{E^{c}} T_{E}^{n} g\right) \rightarrow 0$ as in the proof of Lemma 2. Hence the decomposition $\Theta_{E} g=\Theta_{E} T_{E}^{n} g=\Theta_{E}\left(\chi_{E} T_{E}^{n} g\right)+\Theta_{E}\left(\chi_{E} T_{E}^{n} g\right)$ shows that $\left\|\theta_{E}\right\|_{\infty}=\left\|\chi_{E} \theta_{E}\right\|_{\infty}$. Now, for $n, m \geqq 1$,

$$
\begin{aligned}
\Theta_{E} g & =\Theta_{E} T_{E}^{n} T^{m} g=\lim _{m \rightarrow \infty} \lim _{n \rightarrow \infty} \Theta_{E}\left(\chi_{E} T_{E}^{n} T^{m} g\right) \leqq \lim _{m \rightarrow \infty} \lim _{n \rightarrow \infty}\left\|\theta_{E}\right\|_{\infty}\left\|\chi_{E} T_{E}^{n} T^{m} g\right\|_{1} \\
& =\lim _{m \rightarrow \infty}\left\|\theta_{E}\right\|_{\infty} \Psi_{E} T^{m} g=\left\|\theta_{E}\right\|_{\infty} \Theta_{E} g
\end{aligned}
$$

which completes the proof of the first part, since $\left\|\theta_{E}\right\|_{\infty} \leqq 1$. For the second part, we have, if $g \in L_{1}^{+}, 0 \leqq \Theta_{E F}\left(\chi_{E^{c}} T_{E}^{n} g\right) \leqq \Theta_{E}\left(\chi_{E} T_{E}^{n} g\right) \rightarrow 0$ as $n \rightarrow \infty$ which shows that $\left\|\theta_{E F}\right\|_{\infty}=\left\|\chi_{E} \theta_{E F}\right\|_{\infty}$. Now

$$
\Theta_{E} g-\Theta_{E}\left(\chi_{E} T_{E}^{n} g\right)=\Theta_{E}\left(\chi_{E} T_{E}^{n} g\right) \leqq \Theta_{E \cup F}\left(\chi_{E} T_{E}^{n} g\right) \leqq\left\|\chi_{E} T_{E}^{n} g\right\|_{1} \leqq \Psi_{E} g ;
$$


thus, $\Theta_{E} g \leqq \lim _{n \rightarrow \infty} \Theta_{E \cup F}\left(\chi_{E} T_{E}^{n} g\right) \leqq \Psi_{E} g$. Replacing $g$ by $T^{m} g$ and letting $m \rightarrow \infty$ we get

$$
\Theta_{E} g=\lim _{m \rightarrow \infty} \lim _{n \rightarrow \infty} \Theta_{E \cup F}\left(\chi_{E} T_{E}^{n} T^{m} g\right)
$$

Next, consider

$$
\Theta_{E F}\left(\chi_{E} T_{E}^{n} T^{m} g\right)=\left(\Theta_{E}+\Theta_{F}-\Theta_{E \cup F}\right)\left(\chi_{E} T_{E}^{n} T^{m} g\right)
$$

and let $n \rightarrow \infty$ to get

$$
\Theta_{E F} g=\Theta_{E} g+\lim _{n \rightarrow \infty} \Theta_{F}\left(\chi_{E} T_{E}^{n} T^{m} g\right)-\lim _{n \rightarrow \infty} \Theta_{E \cup F}\left(\chi_{E} T_{E}^{n} T^{m} g\right) .
$$

Now, letting $m \rightarrow \infty$ we have

$$
\Theta_{E F} g=\lim _{m \rightarrow \infty} \lim _{n \rightarrow \infty} \Theta_{F}\left(\chi_{E} T_{E}^{n} T^{m} g\right)
$$

But

Hence

$$
\begin{aligned}
\Theta_{E F} g & \leqq\left\|\theta_{E F}\right\|_{\infty} \lim _{m \rightarrow \infty} \lim _{n \rightarrow \infty}\left\|\chi_{E} T_{E}^{n} T^{m} g\right\|_{1} \\
& \leqq\left\|\theta_{E F}\right\|_{\infty} \lim _{m \rightarrow \infty} \Psi_{E} T^{m} g \\
& \leqq\left\|\theta_{E F}\right\|_{\infty} \Theta_{E} g .
\end{aligned}
$$

$$
\begin{aligned}
\Theta_{E F} g & =\Theta_{E F}\left(T_{E}^{n} T^{m} g\right) \\
& =\lim _{m \rightarrow \infty} \lim _{n \rightarrow \infty} \Theta_{E F}\left(\chi_{E} T_{E}^{n} T^{m} g\right) \\
& \leqq\left\|\theta_{E F}\right\|_{\infty} \lim _{m \rightarrow \infty} \lim _{n \rightarrow \infty} \Theta_{F}\left(\chi_{E} T_{E}^{n} T^{m} g\right) \\
& \leqq\left\|\theta_{E F}\right\|_{\infty} \Theta_{E F} g .
\end{aligned}
$$

This completes the proof, since $\left\|\theta_{E F}\right\|_{\infty} \leqq 1$.

Definition 4. $\Sigma=\left\{E \in \mathscr{F} \mid \Theta_{E E^{c}}=0\right\}$.

LEMMA 5. $\Sigma$ is a field.

Proof. Let $E, F \in \Sigma$ and $G=E \cap F$. Then

$$
0 \leqq \theta_{G G^{c}}=\theta_{G\left(E^{c} \cup F^{c}\right)} \leqq \theta_{G E^{c}}+\theta_{G F^{c}} \leqq \theta_{E E^{c}}+\theta_{F F^{c}}=0 .
$$

Thus $G \in \Sigma$.

Definition $5 . \mathscr{A}$ is the $L_{\infty}$-closure of the class of $\Sigma$-simple functions.

We note that $\mathscr{A}$ is a sub-Banach space of $L_{\infty}$.

THEOREM 1. For a real valued function $f \in L_{\infty}$, the following conditions are equivalent:

(i) $f \in \mathscr{A}$,

(ii) $\lim _{g>g_{0}} \int f g d \mu$ exists for all $g_{0} \in L_{1}^{+}$,

(iii) for all real numbers $\alpha$ and $\varepsilon>0$,

$$
\theta_{E F}=0 \quad \text { where } \quad E=\{x \mid f(x) \leqq \alpha\}, \quad F=\{x \mid f(x) \geqq \alpha+\varepsilon\} .
$$


Proof. (i) $\Rightarrow$ (ii). If $E \in \Sigma$ then $\theta_{E}+\theta_{E^{c}}=\theta_{X}$; thus, for a real valued $g_{0} \in L_{1}^{+}$,

$$
\begin{aligned}
\underset{g>g_{0}}{\limsup } \int_{E} g d \mu & =\underset{g \succ g_{0}}{\lim \sup } \int g d \mu-\limsup _{g>g_{0}} \int_{E^{c}} g d \mu \\
& =\liminf _{g>g_{0}} \int_{E} g d \mu .
\end{aligned}
$$

Therefore $\lim _{g \succ g_{0}} \int \chi_{E} g d \mu$ exists for all $E \in \Sigma$.

Hence it exists for all $\Sigma$-simple functions, and thus for all $f \in \mathscr{A}$.

(ii) $\Rightarrow$ (iii). Suppose $E$ and $F$ are as in (iii) but that $\theta_{E F} \neq 0$. Then $\left\|\theta_{E F}\right\|_{\infty}=1$ and for all $\delta>0$ there exists $g_{0} \in L_{1}^{+}$with $\left\|g_{0}\right\|_{1}=1$ and $\int \theta_{E F} g d \mu \geqq 1-\delta$. Hence $\Theta_{E} g_{0} \geqq 1-\delta$ and $\Theta_{F} g_{0} \geqq 1-\delta$. Thus lim $\sup _{g \succ g_{0}} \int f g d \mu \geqq(1-\delta)(\alpha+\varepsilon)$ and $\lim \inf _{g \succ g_{0}} \int f g d \mu \leqq$ $(1-\delta) \alpha+\delta\|f\|_{\infty}$. If $\delta$ is chosen sufficiently small we see that $\lim _{g>g_{0}} \int f g d \mu$ does not exist.

(iii) $\Rightarrow$ (i). Let $a_{1}<a_{2}<\cdots<a_{n}$ be $n$ numbers and let $E_{i}=\left\{x \mid f(x) \leqq a_{i}\right\}$. Now

$$
\begin{aligned}
\sum_{i=1}^{n} \theta_{E_{i} E_{i}^{c}} & =\sum_{i=1}^{n}\left(\theta_{E_{i}}+\theta_{E_{i}^{c}}-\theta_{X}\right) \\
& \leqq \sum_{i=2}^{n}\left(\theta_{E_{i-1}}+\theta_{E_{i}^{c}}-\theta_{E_{i-1} \cup E_{i}^{c}}\right)+\left(\theta_{E_{n}}+\theta_{E_{1}}{ }^{c}-\theta_{X}\right) \\
& \leqq 1 .
\end{aligned}
$$

Hence if $E_{a}=\{x \mid f(x) \leqq a\}$ then $\theta_{E_{a} E_{a}^{c}} \neq 0$ for only countably many $a$ 's, and so $f \in \mathscr{A}$.

\section{Invariant functions.}

Definition 6. $\mathscr{H}=\left\{f \mid f \in L_{\infty}, f=U f\right\}$ is the class of invariant functions of $U$. We assume $\mathscr{H} \neq\{0\}$.

Note that $\mathscr{H}$ is a sub-Banach space of $L_{\infty}$. Also, if $h \in \mathscr{H}$ and $g^{\prime} \succ g \in L_{1}^{+}$, then $\int h g^{\prime} d \mu=\int h g d \mu$ and hence $\lim _{g^{\prime} \succ g} \int h g^{\prime} d \mu$ exists. Thus $\mathscr{H} \subset \mathscr{A}$.

If $f \in \mathscr{A}$, then $\lim _{n \rightarrow \infty} \int f T^{n} g d \mu=\lim _{n \rightarrow \infty} \int U^{n} f g d \mu$ exists for all $g \in L_{1}(X, \mathscr{F}, \mu)$. Hence the bounded sequence $U^{n} f, n=1,2, \ldots$ has a limit $\pi(f)$ in the $w^{*}$-topology of $L_{\infty}$. Obviously the limit lies in $\mathscr{H}$, so $\pi: \mathscr{A} \rightarrow \mathscr{H}$ is a positive linear contraction.

Definition 7. $\mathscr{A}_{0}=\operatorname{ker} \pi=\left\{f \in \mathscr{A} \mid w^{*}-\lim U^{n} f=0\right\}$. Hence $\mathscr{A} / \mathscr{A}_{0} \cong \mathscr{H}$ is a canonical, isometric isomorphism.

Now $\mathscr{A}$ is a $C^{*}$-algebra with the usual operations. We show that $\mathscr{A}_{0}$ is a closed ideal.

TheOREM 2. $\mathscr{A}_{0}$ is a closed ideal in $\mathscr{A}$.

Proof. Let $f \in \mathscr{A}_{0}$ and assume that $f$ is real. Choose $\varepsilon>0$ and set $E=\{x \mid f(x) \geqq \varepsilon\}$. We may assume $E \in \Sigma$. Suppose $\theta_{E} \neq 0$; then for all $\delta>0$, there is a $g \in L_{1}^{+}$such that $\|g\|_{1}=1$ and $\Theta_{E} g \geqq 1-\delta$. Hence:

$$
\begin{aligned}
0=\lim _{n \rightarrow \infty} \int U^{n} f \cdot g d \mu & =\lim _{n \rightarrow \infty} \int f T^{n} g d \mu \\
& \geqq \varepsilon \lim _{n \rightarrow \infty} \int_{E} T^{n} g d \mu-\|f\|_{\infty} \lim _{n \rightarrow \infty} \int_{E^{c}} T^{n} g d \mu \\
& \geqq \varepsilon(1-\delta)-\|f\|_{\infty} \delta .
\end{aligned}
$$


Clearly, this fails for small $\delta$, and so $\theta_{E}=0$. Thus if $E=\{x|| f(x) \mid>\varepsilon\}$, we have $\theta_{E}=0$.

Now if $h \in \mathscr{A}, h \neq 0$, set $F=\{x|| f(x) h(x) \mid \geqq \varepsilon\}$. Since $F \subset\left\{x|| f(x) \mid \geqq \varepsilon /\|h\|_{\infty}\right\}$, we have $\theta_{F}=0$. Hence

$$
\begin{aligned}
\left|\lim _{n \rightarrow \infty} \int U^{n}(f h) g d \mu\right| & \leqq \varepsilon \lim _{n \rightarrow \infty} \int_{F} T^{n} g d \mu+\varepsilon\|g\|_{1} \quad \text { if } g \in L_{1}^{+} \\
& \leqq \varepsilon\|g\|_{1} \quad \text { for all } \varepsilon>0 .
\end{aligned}
$$

Hence $f h \in \mathscr{A}_{0}$.

As a result of the lemma, we have given $\mathscr{A} / \mathscr{A}_{0}$, and hence $\mathscr{H}$ the structure of a $C^{*}$-algebra. Thus $\mathscr{H}$ has a representation as the set of complex valued continuous functions on its maximal ideal space. This corresponds to Feller's representation [10] of the invariant functions of certain Markov processes, and we shall refer to $\mathscr{H}$ 's maximal ideal space as the Feller boundary.

As is known [8], [11], the Feller boundary is larger than it need be. In the next section, we obtain some properties of ratio ergodic limits, and use them to define a sub $C^{*}$-algebra $\mathscr{G}$ of $\mathscr{H}$, with a maximal ideal space $\mathscr{M}$, smaller than the Feller boundary, but large enough to represent $\mathscr{H}$ as a function algebra on $\mathscr{M}$. This corresponds to the Martin-Doob representation [12], [8], [11] for some classes of functions, and $\mathscr{M}$ will be referred to as the Martin-Doob boundary.

4. Properties of ratio ergodic limits. In [6] Chacon and Ornstein proved that for any $f, g \in L_{1}$, with $g>0$, the limit:

$$
\lim _{n \rightarrow \infty} \frac{\sum_{k=1}^{n} T^{k} f}{\sum_{k=1}^{n} T^{k} g}
$$

exists a.e. We denote the limit function by $(f / g)$. It is also known [5], [4], [1], that if $\alpha \leqq(f / g) \leqq \beta$ a.e. on $E \in \mathscr{F}$, then $\alpha \leqq \Psi_{E}(f) / \Psi_{E}(g) \leqq \beta$.

THEOREM 3. If $f, g \in L_{1}^{+}$with $g>0$, and

$$
\begin{aligned}
& E=\{x \mid(f / g)(x) \leqq a\}, \\
& F=\{x \mid(f / g)(x) \geqq a+\varepsilon\},
\end{aligned}
$$

then $\theta_{E, F}=0$, for all $a \geqq 0$ and $\varepsilon>0$.

Proof. If $\theta_{E, F} \neq 0$ then $\left\|\chi_{E} \theta_{E, F}\right\|_{\infty}=1$. Let $\delta>0$ and set $E_{\delta}=\left\{x \mid \theta_{E, F}(x) \geqq 1-\delta\right\} \cap E$, and similarly for $F_{\delta}$. Then $\left\|\chi_{E-E_{\delta}} \theta_{E-E_{\delta}}, F\right\|_{\infty} \leqq\left\|\chi_{E-E_{0}} \theta_{E, F}\right\|_{\infty} \leqq 1-\delta$. Hence $\theta_{E-E_{\delta}, F}$ $=0$, and so $\theta_{E_{\delta}, F} \leqq \theta_{E, F} \leqq \theta_{E_{\delta}, F}+\theta_{E-E_{\delta}, F}=\theta_{E_{\delta}, F}$ which implies $\theta_{E_{\delta}, F}=\theta_{E, F}$. Now $\psi_{E_{\delta}} \geqq \theta_{E_{\delta} F_{\delta}} \geqq 1-\delta$ on $E_{\delta} \cup F_{\delta}$. Hence $\psi_{E_{\delta}} \geqq(1-\delta) \psi_{F_{\delta}}$ on $E_{\delta} \cup F_{\delta}$, which by Lemma 2 yields $\psi_{E_{\delta}} \geqq(1-\delta) \psi_{F_{\delta}}$, and $\psi_{F_{\delta}} \geqq(1-\delta) \psi_{E_{\delta}}$. Now $(f / g) \leqq a$ on $E_{\delta}$ yields $\Psi_{E_{\delta}} f / \Psi_{E_{\delta}} g \leqq a$. Similarly $(f / g) \geqq a+\varepsilon$ on $F_{\delta}$ implies $\Psi_{F_{\delta}} f / \Psi_{F_{\delta}} g \geqq a+\varepsilon$. These relations yield $a \Psi_{E_{\delta}} g$ $\geqq(1-\delta)^{2}(a+\varepsilon) \Psi_{E_{\delta}} g$ which is false for small $\delta$ if $\Psi_{E_{\delta}}(g) \neq 0$. Hence $\theta_{E, F}=0$. 
Corollary. If $f, g \in L_{1}$ and $(f / g) \in L_{\infty}$, then $(f / g) \in \mathscr{A}$.

Remark. If $T$ is conservative, then Theorem 3 corresponds to the fact that $(f / g)$ is measurable with respect to the $\sigma$-field of invariant sets (cf. [5], [2]).

THEOREM 4. If $(f / g) \in L_{\infty}$, and $h \in \mathscr{A}$, then $\int \pi(h) \cdot f d \mu=\int \pi(h(f / g)) g d \mu$.

Proof. Recall that $\int \pi(h(f / g)) g d \mu=\lim _{n \rightarrow \infty} \int h(f / g) T^{n} g d \mu$. We may assume $f$ and $h$ are real. Choose $\varepsilon>0$, and let $E_{i j}, 1 \leqq i, j \leqq k$ be a $\sum$ partition of $X$ such that

$$
\left\|h-\sum_{i j} h_{i} \chi_{E_{i j}}\right\|_{\infty}<\varepsilon,\left\|(f / g)-\sum_{i j} \alpha_{j} \chi_{E_{i j}}\right\|_{\infty}<\varepsilon
$$

for suitable real $h_{i}, \alpha_{j}$ with $\left|h_{i}\right| \leqq\|h\|_{\infty},\left|\alpha_{j}\right| \leqq\|(f / g)\|_{\infty}$. Now

$$
\begin{aligned}
\left|\lim _{n \rightarrow \infty} \int h(f / g) T^{n} g d \mu-\sum_{i j=1}^{k} h_{i} \alpha_{j} \lim _{n \rightarrow \infty} \int_{E_{i j}} T^{n} g d \mu\right| \\
=\left|\lim _{n \rightarrow \infty} \int h(f / g) T^{n} g d \mu-\sum_{i j} h_{i} \alpha_{j} \Theta_{E_{i j}}(g)\right| \leqq \varepsilon\|g\|_{1}\left(\|h\|_{\infty}+\|(f / g)\|_{\infty}\right) .
\end{aligned}
$$

Let $\delta>0$ be fixed and set $E_{i j}^{\prime}=\left\{x \mid \theta_{E_{i j}}(x) \geqq 1-\delta\right\} \cap E_{i j}$. Then, as before, $\theta_{E_{i j}^{\prime}}=\theta_{E_{i j}}$, and from Lemma $3, \theta_{E_{i j}^{\prime}} \geqq(1-\delta) \psi_{E_{i j}^{\prime}}$. Now $\left|\alpha_{j}-(f / g)\right| \leqq \varepsilon$ on $E_{i j}^{\prime}$ implies that $\left|\alpha_{j}-\Psi_{E_{i j}^{\prime}} f / \Psi_{E_{i j}^{\prime}} g\right| \leqq \varepsilon$. [Here we consider only those $E_{i j}$ 's with $\theta_{E_{i j}} \neq 0$.] Hence:

$$
\left|\sum_{i j} h_{i} \alpha_{j} \Theta_{E_{i j}} g-\sum_{\theta_{E_{i j}} \neq 0} h_{i} \frac{\Psi_{E_{i j}^{\prime}} f}{\Psi_{E_{i j}^{\prime}} g} \Theta_{E_{i},} g\right| \leqq \varepsilon\|h\|_{\infty}\|g\|_{1} .
$$

Also:

$$
\left|\sum_{\theta_{E_{i j}} \neq 0} h_{i} \frac{\Psi_{E_{i j}^{\prime}} f}{\Psi_{E_{i j}^{\prime}} g} \Theta_{E_{i j}} g-\sum_{i j} h_{i} \Psi_{E_{i j}^{\prime}} f\right| \leqq\|g\|_{1}\|h\|_{\infty}\left(\|(f / g)\|_{\infty}+\varepsilon\right) k^{2} \delta .
$$

Finally,

$$
\left|\sum_{i j} h_{i} \Psi_{E_{i j}^{\prime}} f-\sum_{i j} h_{i} \Theta_{E_{i j}} f\right| \leqq\|h\|_{\infty}\|f\|_{1} k^{2} \delta
$$

and

$$
\left|\sum_{i j} h_{i} \Theta_{E_{i j}} f-\lim _{n \rightarrow \infty} \int h T^{n} f d \mu\right| \leqq \varepsilon\|f\|_{1} .
$$

Putting together all these inequalities, we conclude the result.

\section{A representation for $\mathscr{H}$.}

Definition $8 . \mathscr{G}$ is the sub- $C^{*}$-algebra of $\mathscr{H}$ generated by the class $\left\{\pi(l / 1) \mid l \in L_{\infty}\right\}$.

Let $\mathscr{M} \subset \mathscr{G}^{*}$ be the maximal ideal space of $\mathscr{G}$ with the $w^{*}$ topology induced from $\mathscr{G} *$. Let $\mathscr{B}$ be the $\sigma$-field of Baire sets of $\mathscr{M}$.

Note that $\mathscr{G}$ contains the unit $\pi(1)$ of $\mathscr{H}$, and that $g \in \mathscr{G}$ is invertible in $\mathscr{G}$ if and only if it is invertible in $\mathscr{H}$. 
The $C^{*}$-algebra $\mathscr{C}(\mathscr{M})$ of continuous complex valued functions on $\mathscr{M}$ is isometrically* isomorphic to $\mathscr{G}$ under the Gelfand mapping $\sigma: \mathscr{G} \rightarrow \mathscr{C}(\mathscr{M})$. This mapping is order preserving. To see this first we need a few lemmas.

LEMMA 6. If $f \in \mathscr{H}$ then $\pi|f|^{2} \geqq|f|^{2}$.

Proof. We can assume that $f$ is real. Let $g \in L_{1}^{+}$with $\|g\|_{1}=1$. Then

$$
\left|\int f g d \mu\right|=\left|\int f \cdot T g d \mu\right| \leqq\left.\left.\left|\int\right| f\right|^{2} T g d \mu\right|^{1 / 2}\left|\int T g d \mu\right|^{1 / 2}
$$

Hence $\left|\int f g d \mu\right|^{2} \leqq \int U|f|^{2} g d \mu$. If $|f|^{2}>|U f|^{2}$ on a set of positive measure, then there exist $E \in \mathscr{F}, \mu(E)>0, a \geqq 0$ and $\varepsilon>0$ such that $|f| \geqq a+\varepsilon$ and $U|f|^{2} \leqq a^{2}$ on $E$. Take $g=f \chi_{E} /|f| \mu(E)$. Then

$$
(a+\varepsilon)^{2} \leqq\left|\int f g d \mu\right|^{2} \leqq \int U|f|^{2} g d \mu \leqq a^{2}
$$

which is a contradiction. Hence $U|f|^{2} \geqq|f|^{2}$ and $\pi|f|^{2} \geqq|f|^{2}$.

There is a canonical map $j: L_{1} \rightarrow \mathscr{G}^{*}$ defined by $(j f)(g)=\int f g d \mu, f \in L_{1}, g \in \mathscr{G}$. We now show that

LEMmA 7. $\mathscr{M}$ is contained in the $w^{*}$-closure of $j L_{1}^{+}$in $\mathscr{G}^{*}$.

Proof. Choose $m \in \mathscr{M}$ and suppose that the $w^{*}$ neighborhood $\left\{F|| F g_{i}-m g_{i} \mid<\varepsilon\right.$, $i=1, \ldots, n\}$ of $m$ defined by $g_{1}, \ldots, g_{n} \in \mathscr{G}, \varepsilon>0$ is disjoint of $j L_{1}^{+}$. Let

$$
u=\sum_{i=1}^{n} \pi\left[\left(g_{i}-1 m g_{i}\right) \overline{\left(g_{i}-1 m g_{i}\right)}\right] .
$$

Now, let $f \in L_{1}^{+},\|f\|_{1}=1$. Then

$$
\begin{aligned}
\text { (jf }) u & =\sum_{i=1}^{n} \int \pi\left|g_{i}-1 m g_{i}\right|^{2} f d \mu \\
& \geqq \sum_{i=1}^{n} \int\left|g_{i}-1 m g_{i}\right|^{2} f d \mu \\
& \geqq \sum_{i=1}^{n}\left|\int\left(g_{i}-1 m g_{i}\right) f d \mu\right| \geqq \varepsilon^{2} .
\end{aligned}
$$

Hence $u \geqq \varepsilon^{2}$ a.e. and hence $u$ is invertible in $L_{\infty}$. This implies that $u$ is invertible in $\mathscr{G}$. But this is impossible since $m u=0$.

COROLlaRY. $j L_{1}$ is dense in $\mathscr{G}^{*}$ in the $w^{*}$-topology.

THEOREM 5. The Gelfand mapping $\sigma: \mathscr{G} \rightarrow \mathscr{C}(\mathscr{M})$ is positive.

Proof. If $g \geqq 0$ a.e. then $g^{* *} \geqq 0$ on $j L_{1}^{+}$where $g \rightarrow g^{* *}$ is the canonical embedding of $\mathscr{G}$ into $\mathscr{G}^{* *}$. Since $j L_{1}^{+}$is dense in $\mathscr{M}$ and $g^{* *}$ is continuous, $g^{* *} \geqq 0$ on $\mathscr{M}$. Hence $\sigma g=\left.g^{* *}\right|_{\mathscr{M}} \geqq 0$. 
Now we would like to extend $\sigma$ to $\mathscr{H}$. First note that, by the Riesz representation theorem, any $F \in \mathscr{G}^{*}$ can be represented by a measure $\mu_{F}$ on $(\mathscr{M}, \mathscr{B})$. In particular, let $\tilde{\mu}=\mu_{j 1}$. From the order-preserving property of the Riesz representation one can see that for any $f \in L_{1}, \mu_{j f}$ is absolutely continuous with respect to $\tilde{\mu}$. In fact we can obtain $d \mu_{j f} / d \tilde{\mu}$ as follows. First, considering only $L_{\infty}$ functions we have

LEMMA 8. If $f \in L_{\infty}$ then $\mu_{j f} \ll \tilde{\mu}$ and $d \mu_{j f} / d \tilde{\mu}=\sigma \pi(f / 1)$.

Proof. For any $g \in \mathscr{G}$,

$$
\begin{aligned}
\int_{\mathscr{M}} \sigma g \cdot \sigma \pi(f / 1) d \tilde{\mu} & =\int_{\mathscr{M}} \sigma \pi[g \cdot \pi(f / 1)] d \tilde{\mu}=\int_{X} \pi[g \pi(f / 1)] d \mu \\
& =\int_{X} \pi[g(f / 1)] d \mu=\int_{X} g f d \mu,
\end{aligned}
$$

where the last equality follows from Theorem 4.

Definition 9. Let $\tau f=\sigma \pi(f / 1), f \in L_{\infty}$.

Note that the linear mapping $f \rightarrow \tau f$ defines a positive contraction $L_{\infty}(X, \mathscr{F}, \mu)$ $\rightarrow L_{\infty}(\mathscr{M}, \mathscr{B}, \tilde{\mu})$. But it is also a contraction for the corresponding $L_{1}$ norms; hence it is a contraction for all $L_{p}$ norms, $1 \leqq p \leqq \infty$. We can then extend this mapping to $L_{p}(X, \mathscr{F}, \mu) \rightarrow L_{p}(\mathscr{M}, \mathscr{B}, \tilde{\mu})$ with the property that $\int_{X} g f d \mu=\int_{\mathscr{M}} \sigma g \tau f d \tilde{\mu}$ for all $g \in \mathscr{G}, f \in L_{p}$.

We can now prove a representation theorem for $\mathscr{H}$.

THEOREM 6. There is a positive isometric ${ }^{*}$ isomorphism between $\mathscr{H}$ and $L_{\infty}(\mathscr{M}, \mathscr{B}, \tilde{\mu})$.

Proof. Let $h \in \mathscr{H}$ and define $\phi_{h} \in \mathscr{G}^{*}$ by

$$
\phi_{h}(g)=\int_{X} \pi(g h) d \mu .
$$

Note that if $h \in \mathscr{G}$ then $\phi_{h}$ is represented by the measure $\sigma(h) \cdot d \tilde{\mu}$ on $\mathscr{M}$. Let $\gamma_{h}$ be the representing measure of $\phi_{h}, h \in \mathscr{H}$. Then, for any nonnegative continuous function $\sigma g\left(g \in \mathscr{G}^{+}\right)$on $\mathscr{M}$

$$
\left|\int_{\mathscr{M}} \sigma g d \gamma_{h}\right|=\left|\int_{X} \pi(g h) d \mu\right| \leqq\|h\|_{\infty} \int_{X} g d \mu=\|h\|_{\infty} \int_{\mathscr{M}} \sigma g \cdot d \tilde{\mu}
$$

which shows that $\gamma_{h}$ is absolutely continuous with respect to $\tilde{\mu}$ and has a density function bounded by $\|h\|_{\infty}$. We denote this density function by $\sigma h$, noting that it is actually an extension of $\sigma$, and $\|\sigma h\|_{\infty} \leqq\|h\|_{\infty}$. Furthermore, if $l \in L_{\infty}$ then

$$
\begin{aligned}
\int_{\mathscr{M}} \sigma(h) \tau(l) d \tilde{\mu} & =\int_{\mathscr{M}} \sigma(h) \sigma \pi(l / 1) d \tilde{\mu}=\int_{X} \pi(h \cdot \pi(l / 1)) d \mu \\
& =\int_{X} \pi(h \cdot(l / 1)) d \mu=\int_{X} h l d \mu .
\end{aligned}
$$


Hence $\left|\int_{X} h l d \mu\right| \leqq\|\sigma h\|_{\infty}\|\tau l\|_{1} \leqq\|\sigma h\|_{\infty} \cdot\|l\|_{1}$, so $\|h\|_{\infty} \leqq\|\sigma h\|_{\infty}$. Thus the extended $\sigma$ is also an $L_{\infty}$-norm isometry. To show that $\sigma \mathscr{H}=L_{\infty}(\mathscr{M}, \mathscr{B}, \tilde{\mu})$, first note that, if $h \in \mathscr{H}, l \in L_{\infty}(X, \mathscr{F}, \mu)$ then

$$
\left|\int_{X} h l d \mu\right|=\left|\int_{\mathscr{M}} \sigma(h) \tau(l) d \tilde{\mu}\right| \leqq\|\sigma h\|_{1}\|\tau l\|_{\infty} \leqq\|\sigma h\|_{1}\|l\|_{\infty},
$$

hence $\|h\|_{1} \leqq\|\sigma h\|_{1}$. Thus $\sigma^{-1}: \sigma \mathscr{H} \rightarrow \mathscr{H}$ is an $L_{1}$-contraction onto $\mathscr{H}$. Now if $\sigma h_{n}$ is an a.e. monotone sequence in $\sigma \mathscr{H}$ converging a.e. to a function $l$ in $L_{\infty}(\mathscr{M}, \mathscr{B}, \tilde{\mu})$ then $h_{n}$ is an a.e. bounded and monotone sequence in $\mathscr{H}$. If the limit function is $g$, one can easily see that $g \in \mathscr{H}$ and $\sigma g=l$. Since $\sigma \mathscr{H}$ contains the continuous functions, this shows that $\sigma \mathscr{H}=L_{\infty}(\mathscr{M}, \mathscr{B}, \tilde{\mu})$. Now we want to show that

$$
\int_{X} \pi(h f) d \mu=\int_{\mathscr{M}} \sigma(h) \sigma(f) d \tilde{\mu}
$$

for all $h, f \in \mathscr{H}$. In fact, for a fixed $h \in \mathscr{H}$, let $\mathscr{N} \subset \mathscr{H}$ be the class of functions $f$ for which this relation holds. Then $\sigma \mathscr{N}$ contains the continuous functions of $\mathscr{M}$, and one can show, as before, that $\sigma \mathscr{N}$ is closed under a.e. monotone limits. Hence $\sigma \mathscr{N}=L_{\infty}(\mathscr{M}, \mathscr{B}, \tilde{\mu})$.

Finally, we show that extended $\sigma$ is multiplicative, i.e. $\sigma\left(h_{1}\right) \cdot \sigma\left(h_{2}\right)=\sigma\left(\pi\left(h_{1} h_{2}\right)\right)$ for all $h_{1}, h_{2} \in \mathscr{H}$. First note that if $f \in L_{\infty}(\mathscr{M}, \mathscr{F}, \tilde{\mu})$ and $\int_{\mathscr{M}} f \tau(l) d \tilde{\mu}=0$ for all $l \in L_{\infty}(X, \mathscr{F}, \mu)$ then $\sigma^{-1} f=0$, hence $f=0$. Now for $h \in \mathscr{H}, g \in \mathscr{G}, l \in L_{\infty}(X, \mathscr{F}, \mu)$,

$$
\begin{aligned}
\int_{\mathscr{M}} \sigma(h) \sigma(g) \tau(l) d \tilde{\mu} & =\int_{\mathscr{M}} \sigma(h) \sigma(g) \sigma(\pi(l / 1)) d \tilde{\mu} \\
& =\int_{\mathscr{M}} \sigma(h) \sigma \pi(g \pi(l / 1)) d \tilde{\mu}=\int_{X} \pi(h \pi(g \pi(l / 1))) d \mu \\
& =\int_{X} \pi(h g(l / 1)) d \mu=\int_{X} \pi(h g) l d \mu=\int_{\mathscr{M}} \sigma \pi(h g) \tau(l) d \tilde{\mu},
\end{aligned}
$$

hence $\sigma(h) \cdot \sigma(g)=\sigma \pi(h g)$.

Now suppose that $h_{1}, h_{2} \in \mathscr{H}, l \in L_{\infty}(X, \mathscr{F}, \mu)$. Then

$$
\begin{aligned}
\int_{\mathscr{M}} \sigma\left(h_{1}\right) \sigma\left(h_{2}\right) \tau(l) d \tilde{\mu} & =\int_{\mathscr{\mu}} \sigma\left(h_{1}\right) \sigma \pi\left(h_{2} \pi(l / 1)\right) d \tilde{\mu} \\
& =\int_{X} \pi\left(h_{1} \pi\left(h_{2} \pi(l / 1)\right)\right) d \mu=\int_{X} \pi\left(h_{1} h_{2}\right) l d \mu \\
& =\int_{\mathscr{M}} \sigma \pi\left(h_{1} h_{2}\right) \tau(l) d \tilde{\mu}
\end{aligned}
$$

which shows that $\sigma\left(h_{1}\right) \sigma\left(h_{2}\right)=\sigma \pi\left(h_{1} h_{2}\right)$, and completes the proof of the theorem.

We remark that every $f \in L_{p}(\mathscr{M}, \mathscr{B}, \tilde{\mu}), 1 \leqq p<\infty$, induces a function $h \in L_{p}(X, \mathscr{F}, \mu)$, defined by $\int_{X} h l d \mu=\int_{\mathscr{M}} f \tau(l) d \tilde{\mu}$ for all $l \in L_{q}(X, \mathscr{F}, \mu), 1 / p+1 / q=1$. Since $\tau$ is an $L_{q}$-contraction the integral on $\mathscr{M}$ is defined and $h$ satisfies $\int_{X} h l d \mu=$ 
$\int_{X} h T l d \mu$, for all $l \in L_{q}(X, \mathscr{F}, \mu)$. The case $p=1$ causes no difficulty. If $f \in L_{1}(\mathscr{M}, \mathscr{B}, \tilde{\mu}), l \in L_{\infty}(X, \mathscr{F}, \mu)$,

$$
\begin{aligned}
\left|\int_{\mathscr{M}} f \tau(l) d \tilde{\mu}\right| & \leqq\left|\int_{\{|f| \geq n)} f \tau(l) d \tilde{\mu}\right|+\left|\int_{(|f|<n\}} f \tau(l) d \tilde{\mu}\right| \\
& \leqq\|\tau l\|_{\infty} \int_{\{|f| \geq n\}}|f| d \tilde{\mu}+n\|\tau l\|_{1} \\
& \leqq\|l\|_{\infty}\left[\int_{\{|f| \geq n\}}|f| d \tilde{\mu}\right]+n\|l\|_{1} .
\end{aligned}
$$

Thus, if $l_{k}$ is a sequence in $L_{\infty}$ with $\left\|l_{k}\right\|_{1} \rightarrow 0$ and $\left\|l_{k}\right\|_{\infty} \leqq K$ then

$$
\lim _{k}\left|\int f \tau\left(l_{k}\right) d \tilde{\mu}\right| \leqq K \int_{(|f| \geq n\}}|f| d \tilde{\mu} \quad \text { for all } n \geqq 1
$$

Hence this limit is zero and the functional $l \rightarrow \int f \tau(l) d \tilde{\mu}$ on $L$ is induced by an $L_{1}$-function $h$. In a similar way, any Baire measure on $(\mathscr{M}, \mathscr{B})$ induces what one might call "an invariant functional" on $L_{\infty}(X, \mathscr{F}, \mu)$.

We also note the following relation between the maximal ideal spaces of $\mathscr{H}$ and $\mathscr{G}$; that is, between the Feller and Martin boundaries (cf. [9]). Since $\mathscr{H}$ is isometrically isomorphic to $L_{\infty}(\mathscr{M}, \mathscr{B}, \tilde{\mu})$, we state this relation in the following familiar form:

Lemma 9. Let $\mathscr{M}$ be a compact Hausdorff space, $\mathscr{B}$ its Baire sets, and $\tilde{\mu}$ a Baire measure on $(\mathscr{M}, \mathscr{B})$ with support $\mathscr{M}$. Let $\mathscr{M}^{\prime}$ be the maximal ideal space of the $C^{*}$-algebra $L_{\infty}(\mathscr{M}, \mathscr{B}, \tilde{\mu})$. Then there is a continuous and onto map $\rho: \mathscr{M}^{\prime} \rightarrow \mathscr{M}$.

Proof. Interpret $\mathscr{M}^{\prime}$ and $\mathscr{M}$ as classes of homomorphisms and define $\rho: \mathscr{M}^{\prime} \rightarrow \mathscr{M}$ by $\rho(\phi)=\left.\phi\right|_{\mathscr{C}(\mathscr{M})}$. Then $\rho$ is continuous. We show it is onto. Let $m \in \mathscr{M}$, and consider the ideal generated by $m \cdot L_{\infty}(\mathscr{M}, \mathscr{B}, \tilde{\mu})$. If it is proper, it can be embedded in a maximal ideal, whose image must then be $m$ under $\rho$. We show it is proper. If not, then $1=\sum_{1}^{n} f_{i} g_{i}$ where $f_{i} \in m, g_{i} \in L_{\infty}(\mathscr{M}, \mathscr{B}, \tilde{\mu})$. Since $m$ is a maximal ideal, $\exists x_{0}$ such that $f_{i}\left(x_{0}\right)=0 i=1, \ldots, n$. Hence $\left|f_{i}\right| \leqq \varepsilon / h$ sup $\left|g_{i}\right|$ on some neighborhood $U$ of $x_{0}$, such that $\mu(U) \neq 0$. Hence $1=\left|\sum f_{i} g_{i}\right| \leqq \varepsilon$ on $U$, which is a contradiction.

COROLlaRY. $\mathscr{M}$ is homeomorphic to the quotient space $\mathscr{M}^{\prime} / \rho$.

We finish this section by considering the possibility of joining $X$ and $\mathscr{M}$. In general, this cannot be done. If, however, $T$ is induced by a Markov kernel, such that the transform of every point measure is absolutely continuous with respect to $\mu$, then the members of $\mathscr{H}$ can be considered as actual functions on $X$, and the evaluations of these functions at points of $X$ induce bounded linear functionals on $\mathscr{H}$. Hence $X$ can be embedded in $\mathscr{G}^{*}$ (possibly in a many to one fashion). We shall denote the image of $X$ under this mapping as $X$ also. Hence $X \subset j\left(L_{1}^{+}(X, \mathscr{F}, \mu)\right)$. Using the method of Lemma $7, X$ is dense in $\mathscr{M}$, in the $w^{*}$-topology of $\mathscr{G}^{*}$. 
Let $\bar{X}$ be the $w^{*}$-closure of $X$ in $\mathscr{G}^{*}$. Then $\bar{X}$ is a compact Hausdorff space. The following result, stated for the Martin-Doob boundary, is also true for the Feller boundary.

TheOREM 7. For any $g \in L_{1}(X, \mathscr{F}, \mu), T^{n} g d \mu \rightarrow \tau(g) d \tilde{\mu}$ in the $w^{*}$-topology of Baire measures on $\bar{X}$.

Proof. Let $\mathscr{A}_{1}=$ the sub- $C^{*}$-algebra of $\mathscr{A}$, consisting of functions $g^{\prime} \in \mathscr{A}$ such that $\pi\left(g^{\prime}\right) \in \mathscr{G}$.

Let $\mathscr{C}=\left\{f \in \mathscr{C}(\bar{X})|f|_{X} \in \mathscr{A}_{1}\right\}, \mathscr{C}_{0}=\left\{f \in \mathscr{C}(\bar{X})|f|_{X} \in \mathscr{A}_{0}\right\}$. By the Stone-Weierstrass theorem $\mathscr{C}=\mathscr{C}(\bar{X})$. Also, $\mathscr{C}_{0}$ is a closed ideal in $\mathscr{C}$. Let $\mathscr{N} \subset \bar{X}$ be the closed subset such that $\mathscr{C}_{0}=\{f \in \mathscr{C} \mid f(\mathscr{N})=0\}$. Then we have

$$
\mathscr{C}(\mathscr{N}) \cong{ }^{*} \mathscr{C}(\bar{X}) / \mathscr{C}_{0} \cong{ }^{*} \mathscr{A}_{1} / \mathscr{A}_{0} \cong * \mathscr{G} \cong * \mathscr{C}(\mathscr{M})
$$

Hence $\mathscr{C}(\mathscr{N}) \cong{ }^{*} \mathscr{C}(\mathscr{M})$ is induced by a homeomorphism $\phi: \mathscr{N} \rightarrow \mathscr{M}$. Hence $g(s)=g(\phi(s))$ under the above sequence of isomorphisms. But $\mathscr{G}$ separates the points of $\mathscr{G}^{*}$, so $\phi=$ identity and $\mathscr{N}=\mathscr{M}$.

In other words,

$$
\left\{f \in \mathscr{C}(\bar{X})|f|_{X} \in \mathscr{A}_{0}\right\}=\{f \in \mathscr{C}(\bar{X}) \mid f(\mathscr{M})=0\} .
$$

Thus if $f \in \mathscr{C}(\bar{X}), g \in L_{1}(\bar{X}, \mathscr{F}, \mu)$, then:

$$
\int_{X} f T^{n} g d \mu=\int_{X} U^{n}\left(\left.f\right|_{X}\right) g d \mu \rightarrow \int_{X} \pi\left(\left.f\right|_{X}\right) g d \mu=\int_{\mathscr{M}} \sigma \pi\left(\left.f\right|_{X}\right) \tau(g) d \tilde{\mu}
$$

and

$$
\int_{\mathscr{M}} \sigma \pi\left(\left.f\right|_{X}\right) \cdot \tau(g) d \tilde{\mu}=\left.\int_{\mathscr{M}} f\right|_{\mathscr{M}} \cdot \tau(g) d \tilde{\mu}=\int_{X} f \tau(g) d \tilde{\mu}
$$

Thus $T^{n} g d \mu \rightarrow \tau(g) d \tilde{\mu}$.

6. Harmonic functions in the unit disk. As an example we consider a transformation suggested by Feller in [10].

Let $D=\left\{z=\mathrm{re}^{i \phi} \mid 0 \leqq r<1,-\pi \leqq \phi \leqq \pi\right\}$ be the unit disk with the (geometric) boundary $C$. Let $\mathscr{F}$ and $\mu$ be the $\sigma$-field of Borel subsets and the Lebesque measure. For every $z \in D, E \in \mathscr{F}$, let

$$
P(z, E)=\mu\left(Q_{z} \cap E\right) / \mu\left(Q_{z}\right)
$$

where $Q_{z}=\{Z|| Z-z|<1-| z \mid\}$. Then $P$ defines a Markov kernel, such that the transformation of a unit mass at $z \in D$ is given by the measure $P(z, \cdot) \ll \mu$. We let $T$ be the induced transformation on $L_{1}(D, \mathscr{F}, \mu)$. The adjoint $U$ of $T$ is given by

$$
(U f)(z)=\int f(Z) P(z, d Z), \quad f \in L_{\infty}, z \in D .
$$


It is clear that any bounded harmonic function $h$ belongs to $\mathscr{H}$. The converse is also true, but it seems that no explicit proof of it has been given and we would like to indicate an outline for this proof.

If $R$ is a Borel subset of $[0,1)$ let $C_{R}=\{z|| z \mid \in R\}$. One can then obtain the following

Lemma 10. Let $\frac{1}{2} \leqq K<1$ and $R$ be a Borel subset of $[K, 1)$. Then for all $z \in D$, $\frac{1}{2} \leq|z| \leqq K$,

$$
\frac{\mu\left(Q_{z} \cap C_{R}\right)}{\mu\left(Q_{z} \cap C_{[K .1)}\right)} \geqq \frac{1}{16}\left[\frac{\lambda(R)}{1-K}\right]^{3 / 2}
$$

where $\lambda$ is the one dimensional Lebesgue measure.

Corollary. Let $E=C_{[0,1 / 2)} \cup[K, 1)$ and let $f \in L_{1}^{+}, f=0$ a.e. on $C_{[K, 1)}$. Then for all $n \geqq 0$.

$$
\int_{C_{R}} T_{E}^{n} f d \mu \geqq \frac{1}{16}\left[\frac{\lambda(R)}{1-K}\right]^{3 / 2} \int_{C_{(K, 1)}} T_{E}^{n} f d \mu
$$

Using this corollary one can see that if a function $h \in \mathscr{H}$ (which is necessarily continuous) has the form $h\left(\mathrm{re}^{i \phi}\right)=f(r) g(\phi)$ then $\lim _{r \uparrow 1} f(r)$ exists, and that this implies the harmonicity of $h$.

Now if $h$ is any function in $\mathscr{H}$, let $t$ be an irrational number and consider, for a fixed $n,-\infty<n<\infty$,

$$
\lim _{m \rightarrow \infty} \frac{1}{m} \sum_{k=1}^{m}\left(\tau^{k} \cdot z\right)^{-n} h\left(\tau^{k} \cdot z\right)=F_{n}
$$

where $\tau: D \rightarrow D$ is given by $\tau z=e^{i 2 \pi t} z$. This limit $F_{n}$ exists for all nonzero $z \in D$, depends only on $r=|z|$, and satisfies

But, it is clear that

$$
r^{n} F_{n}(r)=\frac{1}{2 \pi} \int_{-\pi}^{+\pi} e^{-i n \phi} h\left(r e^{i n \phi}\right) d \phi
$$

$$
e^{i n \phi} r^{n} F_{n}(r)=\lim _{m \rightarrow \infty} \frac{1}{m} \sum_{k=1}^{m} e^{-i 2 \pi k n t} h\left(r e^{i \Phi} e^{i 2 \pi k t}\right)
$$

is a function in $\mathscr{H}$, hence $e^{i n \phi} r^{n} F_{n}(r)$ must be harmonic, which shows that $r^{n} F_{n}(r)=$ $C_{n} r^{|n|}$ and completes the proof of the following

LemMa 11. A bounded function belongs to $\mathscr{H}$ if and only if it is a harmonic function.

One then shows that the $C^{*}$-algebra $\mathscr{H}$ is isometrically *-isomorphic to $L_{\infty}$ of the unit circle. For any bounded measurable function $l$ on $D$, let $\lambda_{l}$ be the measure on the unit circle obtained by sweeping out $l d \mu$ by the Poisson kernel. The harmonic function $\pi(l / 1)$ corresponds to $d \lambda_{l} / d \lambda$, which is continuous. It then follows that the maximal ideal space $\mathscr{M}$ of $\mathscr{G}$ is homeomorphic to the unit circle. Since $T$ is induced by a Markov kernel, $D$ can be imbedded into $\mathscr{G}^{*}$. Then $D \cup \mathscr{M}$ is homeomorphic to the closed unit disk. 


\section{REFERENCES}

1. M. A. Akcoglu, An ergodic lemma, Proc. Amer. Math. Soc. 16 (1965), 388-392.

2. - Pointwise ergodic theorems, Trans. Amer. Math. Soc. 125 (1966), 296-309.

3. E. Bishop and $\mathrm{K}$. deLeeuw, The representations of linear functionals by measures on sets of extreme points, Ann. Inst. Fourier (Grenoble) 9 (1959), 305-331

4. A. Brunel, Sur un lemme ergodique voisin du lemme de E. Hopf, et sur une des applications, C. R. Acad. Sci. Paris 256 (1963), 5481-5484.

5. R. V. Chacon, Identification of the limit of operator averages, J. Math. Mech. 11 (1962), 961-968.

6. R. V. Chacon and D. S. Ornstein, A general ergodic theorem, Illinois J. Math. 4 (1960), 153-160.

7. G. Choquet, Theory of capacities, Ann. Inst. Fourier (Grenoble) 5 (1955), 131-295.

8. J. L. Doob, Discrete potential theory and boundaries, J. Math. Mech. 8 (1959), 433-458.

9. J. Feldman, Feller and Martin boundaries for countable sets, Illinois J. Math. 6 (1962), 356-366.

10. W. Feller, Boundaries induced by non-negative matrices, Trans. Amer. Math. Soc. 83 (1956), 19-54.

11. G. A. Hunt, Markoff chains and Martin boundaries, Illinois, J. Math. 4 (1960), 313-340.

12. R. S. Martin, Minimal positive harmonic functions, Trans. Amer. Math. Soc. 49 (1941), 137-172.

UNIVERSITY OF TORONTO,

Toronto, Canada 